\section{Quantifying Microbial Activity and Diversity Across Contaminant and Agricultural Gradients In Lower Great Lakes Watersheds}

\author{
NICK W FALK, CHRISTOPHER WEISENER AND \\ KENNETH DROUILLARD
}

University of Windsor

Presenting Author: falkn@uwindsor.ca

Microbial communities play vital roles at the sediment-water interface. They contribute to organic matter degradation, mediate nutrient availability to higher trophic levels, and exert control over $\mathrm{pH}$ and redox gradients. Further, they are often early responders to environmental perturbations and have potential to be integrated into biomonitoring initiatives. However, determining which microbial omic features represent healthy baseline environments and what constitutes a deviation from this state at the community level is still obscure. Thus, focused studies in areas of concern on appropriate temporal and spatial scales are required if microbial data can be deemed a reliable indicator of ecosystem health. Here, a research project within watersheds of the lower Great Lakes of North America was initiated to further understand the community and functional responses of sediment microbial assemblages to anthropogenic chemical and nutrient stressors using a combination of metatranscriptomics as well as amplicon sequencing based on the active (RNA) and potential (DNA) microbial consortia. Sites targeted in the study included the Detroit River, a region with a complex history of industrialization and urbanization, as well as watersheds spanning an agricultural gradient across southern Ontario, Canada. Differential gene expression analysis revealed that microbial function varied from natural to impacted sites. In the Detroit River, legacy-polluted sediments showed increased catabolic and anabolic cycling associated with nitrate reduction, methanogenesis, and beta-oxidation, despite decreases in community $\alpha$-diversity. Transcripts involved in cell gluconeogenesis and polyester synthesis also showed high abundance in contaminated zones, along with increased expression of stress response genes and genetic transfer mechanisms. Microbial activity spanning the agricultural gradient revealed that regions defined by selected fertilizer practices (i.e. chemical fertilizer vs. manure application) were as different from one another in terms of gene expression as they were from natural, unperturbed control sites. Further, assessing diversity through both RNA and DNA metabarcoding allowed for differentiation of the active and dormant microbial taxa. By studying the genetic mechanisms microbial communities employ to moderate the effects of contaminants and nutrient loads, their use as early warning indicators of human-induced landscape change can be better understood.

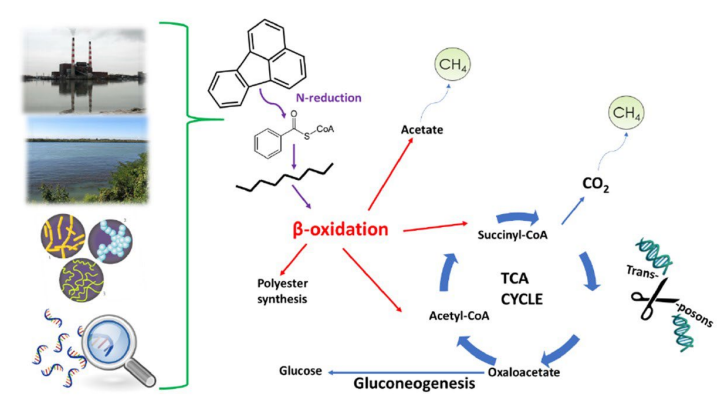

